\section{Prothrombotic state in young females with severe early-onset obesity}

Advance online publication, 25 October 2017; doi:10.1038/pr.2017.248

To the Editor: The prevalence of obesity is increasing globally. Obesity associates with disturbed inflammatory and coagulation systems (1) that are intertwined at many levels. Previous studies indicate various coagulation abnormalities and increased thrombin generation in obese children and adults. We aimed to further explore several measures of coagulation system and their relation to key clinical parameters in a welldefined case-control study of young subjects who had developed severe obesity already in early childhood.

We included subjects whose weight-for-height ratio exceeded $60 \%$ (corresponding to age- and sex-specific body mass index (BMI) $\geq 99$ th percentile) before age 7 years, who were referred to Children's Hospital in Helsinki because of severe obesity, and who were between 15 and 25 years of age at the time of the study $(n=54,27$ females and 27 males; median age 18.8 years; median BMI $41 \mathrm{~kg} / \mathrm{m}^{2}$ ). We recruited from the national population register non-obese controls with similar age range $(n=64,35$ females and 29 males; median age 19.4 years; median BMI $22 \mathrm{~kg} / \mathrm{m}^{2}$ ). Institutional Ethics Committee approved the study and all study participants or their guardians gave an informed written consent. Weight, height, BMI, and waist circumference were measured. Blood samples were collected after an overnight fast; at the time of sampling, all participants were evaluated by a researcher and were found to be free of infections. Samples were analyzed for serum high-sensitive C-reactive protein (hs-CRP), insulin, and leptin. Plasma samples for prothrombin time (PT-\%), activated partial thromboplastin time, thrombin time, fibrinogen, coagulation factor VIII (FVIII) activity, antithrombin activity, and D-dimer were available for a sub-population comprising 46 obese young adults ( 25 females and 21 males) and 38 controls (24 females and 14 males). Prothrombin fragment $\mathrm{F} 1+2$ was analyzed for all subjects. $\mathrm{F} 1+2$ values outside the analyzing range $(n=3)$ were omitted from the analyses. Mann-Whitney $U$-test, Spearman's correlation, and linear regression were used for statistical analyses. As several variables differed between sexes, males and females were analyzed separately. At the time of the study, 3 of 27 obese females (11\%) and 7 of 35 control females (20\%) used oral contraceptives. All the analyses were carried out with and without the oral contraceptive users and the results remained similar. Also, the coagulation markers did not differ between the subjects with and without the use of oral contraceptives (F1+2; $P=0.6$, fibrinogen; $P=0.1$, FVIII; $P=0.4$ ).

Obese subjects had higher insulin, leptin, and hs-CRP concentrations $(P<0.001)$ than control subjects. In obese females, activities of fibrinogen and FVIII were higher $(P<0.05)$, and thrombin time as well as prothrombin time were shorter (e.g. PT-\% was higher) $(P<0.05)$ when compared with control females. Difference in $\mathrm{F} 1+2$ levels did not reach statistical significance in females $(P=0.07)$ (Figure 1). In obese males, fibrinogen activity was higher and thrombin time shorter $(P<0.05)$ than in control males. Antithrombin, D-dimer, and activated partial thromboplastin time did not differ between the obese and control subjects in either sex.

Correlations between markers of coagulation and BMI, waist circumference, hs-CRP, leptin, and insulin were tested. The strongest positive correlations were observed between fibrinogen and BMI, waist circumference, hs-CRP, insulin, and leptin in both sexes $(r>0.6, P<0.001$ in all correlations) (Supplementary Table S1 online). Positive correlations were observed also between hs-CRP and PT-\%, FVIII, and F1+2 in females $(P<0.001)$. Further, multiple linear regression analyses were performed to explore determinants for fibrinogen, FVIII, and F1+2 (Supplementary Table S2). The most powerful determinants for fibrinogen were hs-CRP in both sexes and BMI in females. The model explained $67 \%$ and $71 \%$ of the variance in fibrinogen in females and males, respectively. Insulin and hs-CRP were significant determinants of FVIII in females, but not in males. In females, the model accounted for $23 \%$ of the variance in FVIII.

Young females with early-onset obesity showed a prothrombotic state characterized by elevated levels of fibrinogen and FVIII activity. There was also a trend towards higher F1 +2 levels. The production of fibrinogen can be induced by inflammatory mediators originating from the adipose tissue in obese individuals. The regression analysis confirmed that hsCRP was the most powerful determinant of fibrinogen in all subjects, and BMI was also associated with fibrinogen levels, especially in females. Elevated fibrinogen levels have been associated with cardiovascular complications. Accordingly, an increase of fibrinogen concentration by $1 \mathrm{~g} / \mathrm{l}$ predicts a doubling in the rate of cardiovascular events (2). Elevated fibrinogen is also associated with venous thrombosis; an increase in the fibrinogen levels from 3 to $5 \mathrm{~g} / \mathrm{l}$ has been demonstrated to increase the risk for thrombosis fourfold (3). In our study, the level of fibrinogen exceeded the reference range of $4 \mathrm{~g} / \mathrm{l}$ in $72 \%$ of obese young females, and in $40 \%$ of obese females the level exceeded $5 \mathrm{~g} / \mathrm{l}$, suggesting that there may be an increased risk for both arterial and venous thromboses.

Similar to fibrinogen, production of FVIII can be induced in inflammatory states. In line with this, we observed that 


\section{Letter to the Editor}

a
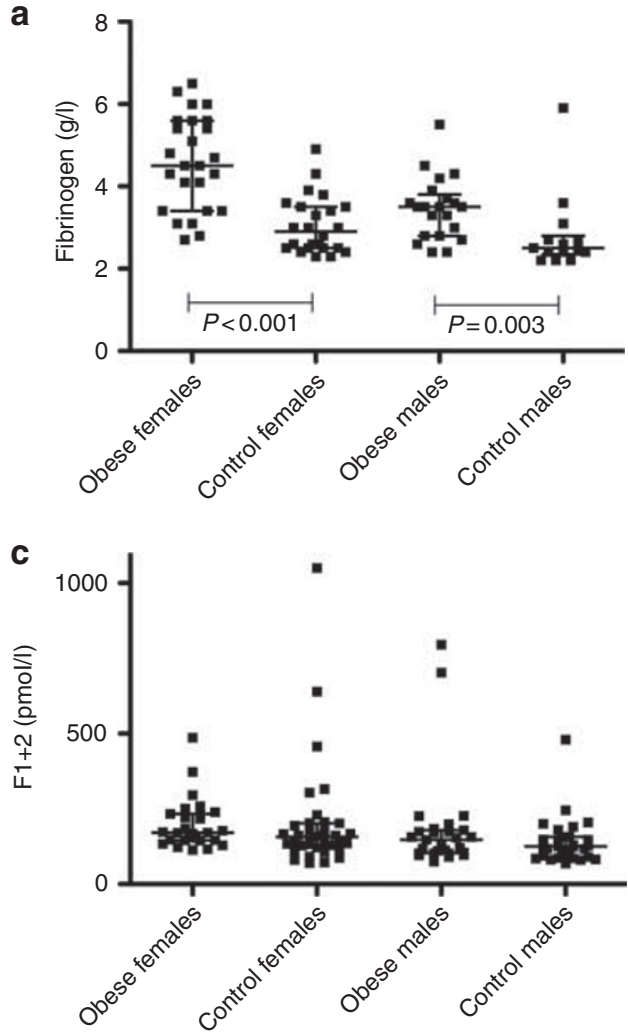

b

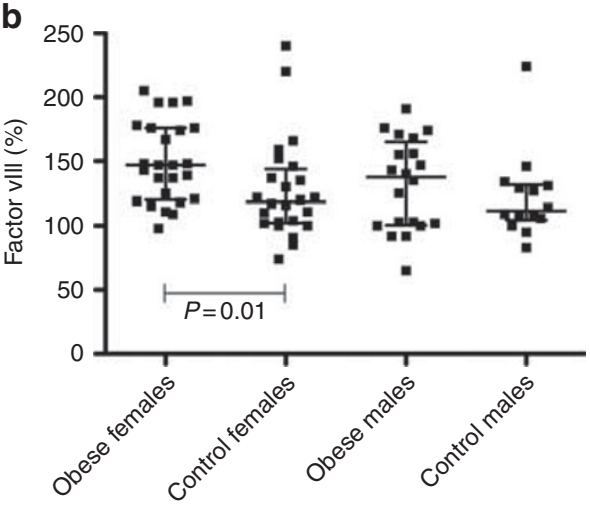

d

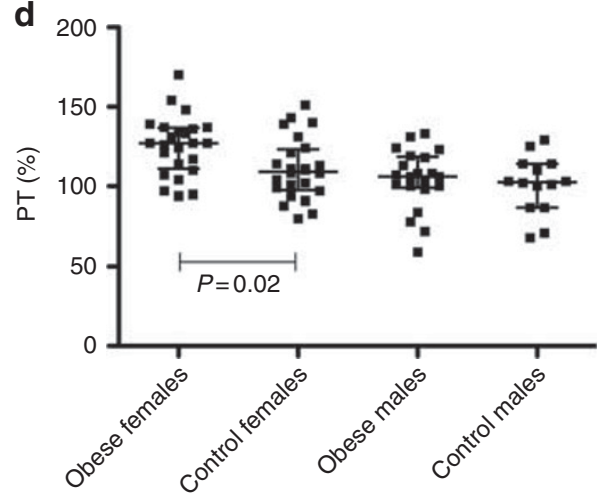

Figure 1. Coagulation variables in obese and control females and males. (a) Fibrinogen, (b) coagulation factor VIII, (c) prothrombin fragment F1+2, and (d) prothrombin time (PT-\%).

hs-CRP was a strong determinant of FVIII activity, particularly in females. Interestingly, in our cohort insulin was the most powerful determinant of FVIII activity in females. Insulin resistance is proposed to impair endothelial function, and in a recent study, it contributed to increased activities of several coagulation factors including FVIII and fibrinogen (4). Furthermore, persistently elevated FVIII has been shown to predict the recurrence of venous thrombosis (5).

Prothrombin fragment F1+2 levels were analyzed given that thrombin has multiple functions in promoting coagulation. There was a trend towards higher F1+2 in obese females, and in females F1+2 correlated with hs-CRP, insulin, and leptin. Mechanisms contributing to these associations may include the stimulation of tissue factor expression via leptin on blood mononuclear cells or its secretion from adipocytes (1).

In the current study, especially young obese females demonstrated signs of coagulation activation known to be related to thrombotic tendency. Even though children are developmentally protected from thrombosis, obesity together with other risk factors may induce a relevant prothrombotic state, which needs to be taken into consideration already at young age when treating obese children and adolescents. Further studies with longitudinal assessments are warranted to elucidate the clinical significance of our findings.
SUPPLEMENTARY MATERIAL

Supplementary material is linked to the online version of the paper at http://www.nature.com/pr

\section{STATEMENT OF FINANCIAL SUPPORT}

This study was financially supported by the Academy of Finland, Sigrid Jusélius Foundation, Foundation for Pediatric Research, Folkhälsan Research Foundation, Swedish Research Council, Swedish Childhood Cancer Foundation, and Helsinki University Hospital research funds.

Disclosure: The authors declare no conflict of interest.

Petra Loid' ${ }^{1}$, Satu Långström ${ }^{1}$, Heli Viljakainen ${ }^{1,2}$, Anne Mäkipernaa ${ }^{3}$, Markku Heikinheimo ${ }^{1}$ and Outi Mäkitie ${ }^{1,2,4}$

${ }^{1}$ University of Helsinki and Helsinki University Hospital, Children's Hospital, Helsinki, Finland;

${ }^{2}$ Folkhälsan Institute of Genetics and University of Helsinki,

Helsinki, Finland;

${ }^{3}$ Children's Hospital and Hematology, Comprehensive Cancer Center, Helsinki University Hospital, University of Helsinki, Helsinki, Finland;

${ }^{4}$ Center for Molecular Medicine, Karolinska Institutet, and Clinical Genetics, Karolinska University Hospital, Stockholm, Sweden.

Correspondence: Outi Mäkitie (outi.makitie@helsinki.fi) 


\section{Letter to the Editor}

\section{REFERENCES}

1. Samad F, Ruf W. Inflammation, obesity, and thrombosis. Blood 2013;122: 3415-22.

2. Danesh J, Lewington S, Thompson SG, et al. Plasma fibrinogen level and the risk of major cardiovascular diseases and nonvascular mortality: an individual participant meta-analysis. JAMA 2005;294:1799-809.

3. Koster T, Rosendaal FR, Reitsma PH, van der Velden PA, Briët E, Vandenbroucke JP. Factor VII and fibrinogen levels as risk factors for venous thrombosis. A case-control study of plasma levels and DNA polymorphismsthe Leiden Thrombophilia Study (LETS). Thromb Haemost 1994;71:719-22.

4. Lallukka S, Luukkonen PK, Zhou Y, et al. Obesity/insulin resistance rather than liver fat increases coagulation factor activities and expression in humans. Thromb Haemost 2017;117:286-94.

5. Timp JF, Lijfering WM, Flinterman LE, et al. Predictive value of factor VIII levels for recurrent venous thrombosis: results from the MEGA followup study. J Thromb Haemost 2015;13:1823-32. 